\title{
Constructing the Spectral Energy Distribution Model of Galaxies Considering Dust Extinction and Re-emission
}

\author{
Atsuko T. Kawakita* \\ Nagoya University \\ E-mail: kawakita.atsukode.mbox.nagoya-u.ac.jp \\ Tsutomu T. Takeuchi \\ Nagoya University \\ E-mail: takeuchi.tsutomudg.mbox.nagoya-u.ac.jp
}

\begin{abstract}
Heavy elements are formed by nucleosynthesis in the process of stellar evolution, and a significant fraction of the amount produced is injected into interstellar space in the form of dust grains. Dust grains scatter and absorb ultraviolet (UV) and optical emission from stars, and re-emit the absorbed energy as infrared (IR) radiation. This process prevents us from directly observing stellar light from galaxies. To understand the intrinsic properties of galaxies from their spectra, we must take into account both dust extinction and re-emission by dust.

In this study, we construct spectral energy distribution (SED) models of galaxies as a function of galactic chemical evolution, taking into account dust extinction and re-emission. We use the evolutionary synthesis code "PEGASE”[四] to obtain stellar spectra of galaxies. We used extinction curves of [[]], [B] and [田], and employ [G] for dust re-emission spectra.

We calculated the evolution of the SEDs of galaxies. These results lead us to three conclusions. First, these SEDs have similar shapes and they behave similarly with time. Second, galaxies can be observed as luminous IR galaxies when galactic age is about from 500 Myr to 1 Gyr. Finally, present-day optically luminous galaxies were bright in IR in previous times.

By using this model we can determine the metallicity in galaxies as a function of galactic age and calculate the dust extinction and re-emission consistently with metallicity. We can estimate the star formation rate, metallicity, dust abundance and other important quantities of high- $z$ galaxies by fitting this model to SEDs observed for high-z sources.
\end{abstract}

The Life Cycle of Dust in the Universe: Observations, Theory, and Laboratory Experiments 18-22 November, 2013

Taipei, Taiwan

\footnotetext{
*Speaker.
} 


\section{Introduction}

Star formation history and chemical evolution are fundamental pieces to understand galaxy evolution. There are many dust-rich regions in the ISM, often coinciding with sites of star formation. Dust grains scatter and absorb ultraviolet (UV) and optical photons from stars, and re-emit the absorbed energy as IR photons. Thus, in the optical, star formation is hidden by dust grains. Therefore, we must consider both dust extinction and re-emission to understand star formation history and more intrinsic properties of galaxies especially when we observe galaxy spectra.

\section{Method}

To model the stellar contribution to the spectra of galaxies, we adopt the evolutionary synthesis code "PEGASE" [四]. This code calculates evolution of the stellar SED of galaxies as a function of star formation history. In this code, the monochromatic flux of a galaxy at age $t$ and wavelength $\lambda$ is calculated by

$$
F_{\lambda}(t)=\int_{0}^{t} \int_{m_{\mathrm{l}}}^{m_{\mathrm{u}}} S(t-\theta) \phi(m) f_{\lambda}(m, \theta) \mathrm{d} m \mathrm{~d} \theta,
$$

where $S(t-\theta)$ is the star formation rate (SFR) at time $t-\theta$ in the unit of $M_{\odot}$ per time and mass units, $\phi(m)$ is the initial mass function (IMF) defined in the interval $\left[m_{1}, m_{\mathrm{u}}\right]$ and normalized to $1 M_{\odot}$, and $f_{\lambda}(m, \theta)$ is the monochromatic flux of a star with initial mass $m$ at wavelength $\lambda$ and age $\theta$ since zero age main sequence (ZAMS). We adopt the following formula to evaluate how much the UV and optical emission decrease:

$$
f_{\text {obs }}=f_{0} 10^{-31.1 Z_{\text {gas }}(t)\left(\frac{A_{\lambda}}{A_{V}}\right)\left(\frac{M_{\text {gas }}(t)}{M_{\text {gal }}(t)}\right)}
$$

Here, $f_{0}$ and $f_{\text {obs }}$ are the emitted and observed flux from stars respectively. $Z_{\text {gas }}$ is the gaseous metallicity at time $t, A_{\lambda}$ and $A_{V}$ the obscuration at wavelength $\lambda$ and $V$ band respectively (in magnitude), and $M_{\mathrm{gas}}(t)$ and $M_{\mathrm{gal}}(t)$ are the mass of gas and the galaxy at time $t$. We can derive eq. 2.2 from the optical thickness of gas radiation from a galaxy calculated by [ $[$ ]

$$
\tau_{\lambda, \mathrm{g}}=3.25\left(\frac{A_{\lambda}}{A_{V}}\right)\left(\frac{Z_{\mathrm{gas}}(t)}{Z_{\odot}}\right)\left(\frac{M_{\mathrm{gas}}(t)}{M_{\mathrm{gal}}(t)}\right)
$$

in the assumption of the uniform screen model [ [8] which is a dust distribution model; dust grains are distributed on the screen uniformly. Here, $Z_{\odot}$ represents the metallicity of the solar system. From Anders \& Grevesse [Q], we use $Z_{\odot}=0.02$. For the dust extinction curve, we quote extinction curves from [], [园] and [团] . These models are depending only on the wavelength and the extinction $R_{V}$.

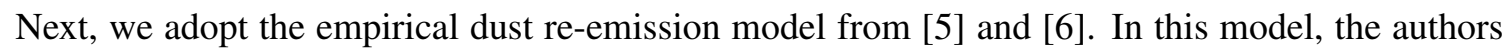
classified dust grains into three populations depending on their size and then synthesized each component of the spectra. In [[5] and [6], Their parameter $\alpha$ quantifies the hardness of the radiation field. For example, a small $\alpha$ represents a large contribution of massive stars. We show these the dust extinction curve models and the dust re-emission model in Fig.1. We calculate theoretical SEDs of galaxies on the assumption that all the absorbed UV and optical photons are re-emitted as IR photons. 

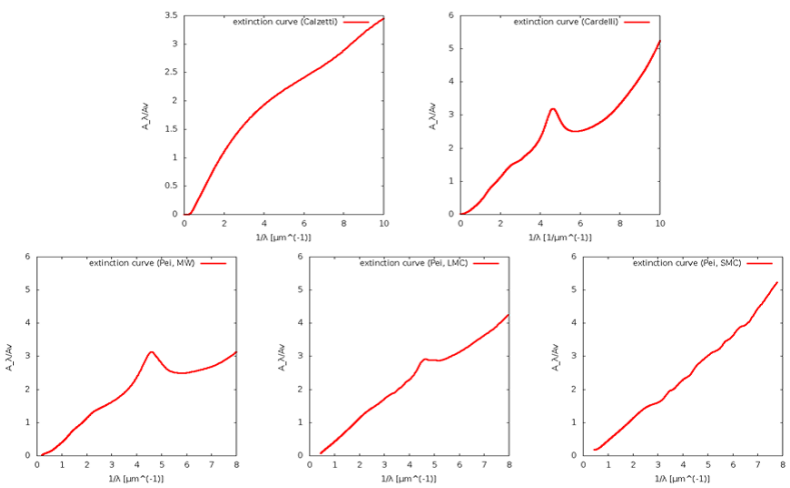

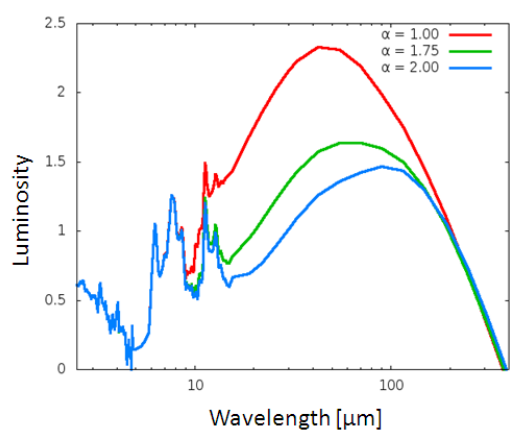

Figure 1: Left panels: Extinction curves. Top left panel: the extinction curve for starburst galaxies from [D], top right panel: for nearby galaxies from [B], bottom panels from left to right: for the Milky Way (MW), the Large Magellanic Cloud (LMC) and the Small Magellanic Cloud (SMC) from [四] respectively. Right panel: Re-emission curve of dust from [ [B] and [ [G]. Here, $\alpha$ is a parameter to quantify the hardness of the radiation field.
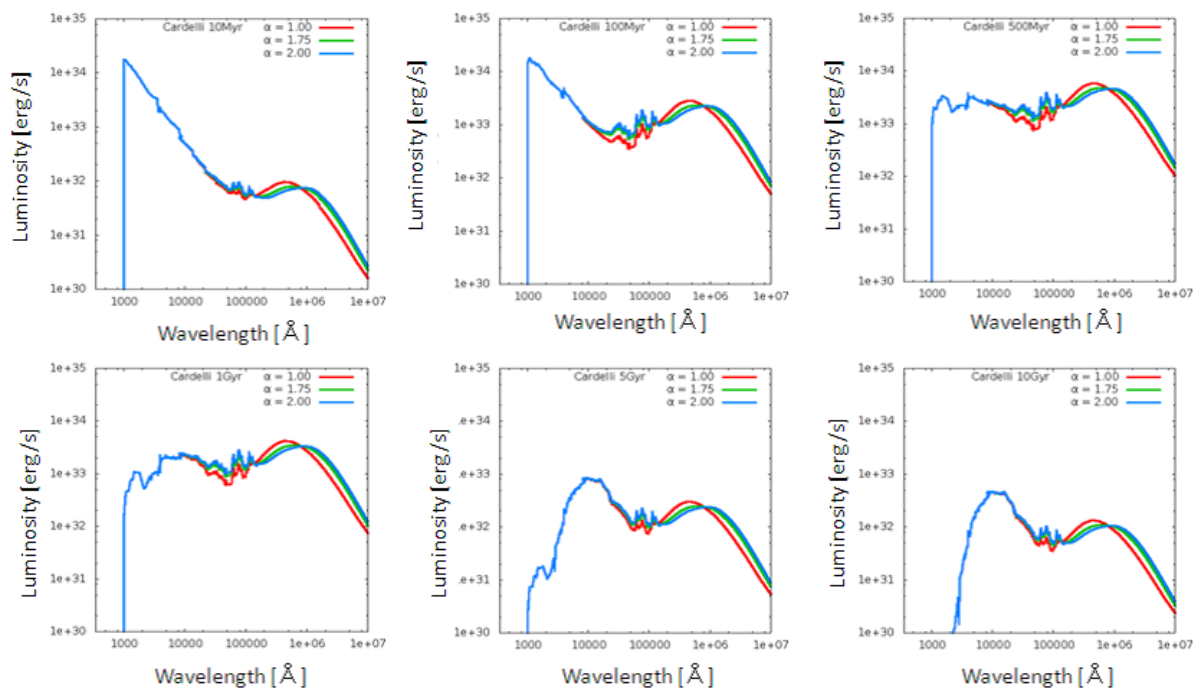

Figure 2: These graphs show the SED evolution. The top plots from left to right: our theoretical SED models at the galactic ages $10 \mathrm{Myr}, 100 \mathrm{Myr}, 500 \mathrm{Myr}$ respectively. The bottom plots: at the galactic ages 1 Gyr, 5 Gyr, 10 Gyr.

\section{RESULTS \& DISCUSSION}

We calculated a grid of SED models with different extinction curves and values of $\alpha$. The results do not depend strongly on the choice of extinction curve, so we show the results using the curve for nearby galaxies [3] only. We calculate the SEDs at different galactic ages to follow the SED evolution and the effect of chemical evolution. At $10 \mathrm{Myr}$, there are not many heavy elements in galaxies because the galaxies are very young. The abundance of dust grains is low, so the SEDs are less affected by dust grains. For these reasons, galaxies are most luminous in the 
UV. At $100 \mathrm{Myr}$, dust grains are gradually forming in galaxies. The SEDs are more affected by dust extinction at this age than at the age of $10 \mathrm{Myr}$ and dust re-emission in the IR becomes more prominent. At $500 \mathrm{Myr}$ and $1 \mathrm{Gyr}$, galaxies are more luminous in the IR than in the UV. Finally, at $5 \mathrm{Gyr}$ and $10 \mathrm{Gyr}$, the UV flux is further decreasing because the massive stars which radiate at UV are already gone. The IR flux is also decreasing because the total amount of interstellar dust decreases with the consumption of gas. Therefore old galaxies are most luminous at optical wavelength.

\section{CONCLUSION}

We can derive three conclusions from above results. First, SEDs derived from different extinction curves have similar shapes and they behave similarly with time. Second, galaxies can be observed as luminous IR galaxies when the galactic age is between $500 \mathrm{Myr}$ and $1 \mathrm{Gyr}$, because re-emission is most enhanced at this age. Finally, present-day optically bright galaxies were bright in the IR in previous times.

Using the model calculations in this study, we can determine the metallicity in galaxies as a function of galactic age and calculate the dust extinction and re-emission consistently with metallicity. From best fit parameters we can estimate the star formation rate, metallicity, dust abundance and other important quantities of high-z galaxies by fitting this model to SEDs observed for high-z galaxies through eq.

\section{References}

[1] M. Fioc and B. Rocca-Volmerange, PEGASE: a UV to NIR spectral evolution model of galaxies, A\&A 326 (1997) 950-962

[2] D. Calzetti, L. Armus, R. C. Bohlin, A. L. Kinney, J. Koornneff and T. Storchi-Bergmann, The Dust Content and Opacity of Actively Star-forming Galaxies, ApJ $\mathbf{5 3 3}$ (2000) 682-695

[3] J. A. Cardelli, G. C. Clayton and J. S. Mathis, The relationship between infrared, optical, and ultraviolet extinction, ApJ 345 (1989) 245-256

[4] Y. C. Pei, Interstellar dust form the Milky Way to the Magellanic Clouds, ApJ 395 (1992) 130-139

[5] D. A. Dale, G. Helou, A. Contursi, N. A. Silbermann and S. Kolhatkar, The Infrared Spectral Energy Distribution of Normal Star-forming Galaxies, ApJ 549 (2001) 215-227

[6] D. A. Dale and G. Helou, The Infrared Spectral Energy Distribution of Normal Star-forming Galaxies: Calibration at Far-Infrared and Bubmillimeter Wavelength, ApJ 576 (2002) 159-168

[7] B. Guiderdoni and B. Rocca-Volmerange, A model of spectrophotometric evolution for high-redshift galaxies, A\&A 186 (1987) 1-21

[8] D. Calzetti, A. L. Kinney and T. Storchi-Bergmann, Dust extinction of the stellar continua in starburst galaxies: The ultraviolet and optical extinction law, ApJ $\mathbf{4 2 9}$ (1994) 582-601

[9] E. Anders and N. Grevesse, Abundances of the elements: Meteoritic and solar, GeCoA 53 (1989) $197-214$ 